www.jmscr.igmpublication.org

Index Copernicus Value: 79.54

ISSN (e)-2347-176x ISSN (p) 2455-0450

crossrefDOI: https://dx.doi.org/10.18535/jmscr/v7i2.04

Journal Of Medical Science And Clinical Research

IGM Publication

An Official Publication of IGM Publication

\title{
Assessment of Chronic Musculoskeletal Pain Management in Elderly Patient at General Hospital in Patna City, India
}

\author{
Author \\ Dr MD. Farid Alam Ansari \\ Department of Medicine, Patna Medical College \& Hospital, Patna \\ Email: firdausinshahasan@gmail.com
}

\begin{abstract}
Back Ground\& Objective: Chronic musculoskeletal pain conditions are a major burden on individuals, health systems, and social care systems, with indirect costs being predominant. This burden has been recognized by the United Nations and WHO, by endorsing the Bone and Joint Decade 2000 2010. The four major musculoskeletal conditions: Gout arthritis, rheumatoid arthritis and low back pain. Rheumatoid arthritis, which is characterized by loss of joint cartilage that leads to pain and loss of function primarily in the knees and hips, affects $9.6 \%$ of men and $18 \%$ of women aged $>60$ years. Increases in life expectancy and ageing populations are expected to make Rheumatoid arthritis the fourth leading cause of disability by the year 2020. Objective of this trial was to assess chronic musculoskeletal pain management in elderly patient at Patna Medical College \& Hospital.

Methodology: A cross sectional study involving patient chart review was conducted to assess chronic musculoskeletal pain management in Patna Medical College \& Hospital from May 1, 2018 to July 30,2018.

Result: From a total of 131 chronic musculoskeletal pain management in elderly patient those registered at Patna Medical College Hospital from May 1,2018 to Jul30,2018, of these 82(62.6\%)were male and 49(37.4\%)were female. This study shows that male were more affected with chronic musculoskeletal pain 82(62.6\%) than female 49(37.4\%) among this with gout arthritis 26(86.6\%) were male and $4(13.3 \%)$ were female, Rheumatoid arthritis $46(58.9 \%)$ were male and 32(41.1\%)were female whereas with low back pain 10(43.5\%)were male and 13(56.5\%)were female.

Conclusion: This study shows, from chronic musculoskeletal pain, Rheumatoid arthritis was more prevalent $78(59.5 \%)$ when compared with other type of chronic musculoskeletal pain in elderly while, low back pain is the least10(43.5\%) and gout arthritis30(22.90\%) have moderate prevalence and chronic musculoskeletal pain was more prevalent in male elderly patient 82 (62.6\%) when compared with female elderly patient $49(37.4 \%)$ as indicated from patient chart which were recorded from May 1,2018 to Jul 30,2018 at Patna Medical College \& Hospital. Since treating elderly patient need great precaution regarding drug metabolism and excretion so prior to prescribing NSAID, Steroid and other anti-pain to treat Chronic MSK pain management in elderly, pertinent lab finding such as Liver function test, Renal function test are essential.

Keywords: Pain Management, Chronic Musculoskeletal, Elderly Patients Rheumatoid arthritis.
\end{abstract}




\section{Introduction}

Chronic musculoskeletal pain conditions are a major burden on individuals, health systems, and social care systems, with indirect costs being predominant. This burden has been recognized by the United Nations and WHO, by endorsing the Bone and Joint Decade 2000- 2010. The four major musculoskeletal conditions: Goutarthritis, rheumatoid arthritis and low back pain. Rheumatoid arthritis, which is characterized by loss ofjoint cartilage that leads to pain and loss of function primarily in the knees and hips, affects $9.6 \%$ of men and $18 \%$ of women aged $>60$ years. Increases in life expectancy and ageing populations are expected to make Rheumatoid arthritis the fourth leading cause of disability by the year 2020..$^{[1,2,3,4]}$ In case of chronic non-cancer pain (involves musculoskeletal condition such as arthritis has been described as a "disease "concept rather than series ofsymptoms. The use of oral medication in the treatment of chronic musculoskeletal pain in the elderly requires careful selection of drugs to control pain with consideration for both the physiological state and the presence of disease(s). Recent advances have improved the understanding of bimolecular mechanisms of chronic pain. These include the production of powerful proinflammatory cytokines by glial and microglia cells, which then lead to activation of major pain pathways from the periphery through the dorsal horn and supraspinal pathways to the somatosensory and other higher cortical centers. This has allowed better recognition for intervention with antiinflammatory agents to control cytokine production (e. g. prednisolone, triamcinolone and other brain-penetrating corticosteroids). Advances in understanding of chronic pain have lead to recognition of neuronal PX2 puringergic receptors as potential sites for drugs to control pain by more selective actions. Cardio-renal effects have been attributed to some anti pain (e. g. diclofenac), but not all (e. g. naproxen) conventional NSAIDs. Here we make recommendations for a selection of certain NSAIDs to be used for pain therapy in the elderly in consideration of their relative safety and pharmacokinetics. While newer formulations of narcotics have given some advance in pain control, the application of this group of drugs requires close supervision in the elderly, especially those with cognitive decline, since drug actions on the central and peripheral nervous systems can result in significant adverse effects of these agents (e. g. constipation, drowsiness, respiratory and cardiovascular decline)..$^{[5,6,7,8]}$

Musculoskeletal (MSK) pain is one of the leading causes of chronic health problems in people over 65 years of age. Studies suggest that a high prevalence of older adults suffer from MSK pain $(65 \%$ to $80 \%) .{ }^{[12]}$ The prevalence of pain in the elderly is not accurately known, some studies suggest that older adults have an even higher prevalence of MSK pain, between $65 \%-85 \%$ with $36 \%$ to $70 \%$ reportedly suffer from a back pain, Rheumatoid arthritis and gout arthritis condition. $^{[13,14]}$

Authors evaluated quality indicators for chronic pain in a random sample of 372 older community dwelling patients using medical record review and interviews. They concluded that chronic pain management in older vulnerable patients is inadequate and that improvement is needed in screening, clinical evaluation, follow up and attention to potential toxicities of therapy. ${ }^{[15]}$

As my knowledge currently there was no adequate study that was done on assessment of chronic musculoskeletal pain management in elderly patient in Patna so that this study will help for members of Patna Medical College \&Hopital to know the magnitude and prevalence of the chronic musculoskeletal pain in elderly patient. Despite the essential role of ant pain in reducing chronic musculoskeletal pain, it remain cause of complication and permanent physical disability in elderly people. Preventing elders from developing chronic musculoskeletal pain in the first is critical to reducing its physical disability. But once an elder develops chronic musculoskeletal pain a care giver must recognize the symptom and seek appropriate management. 


\section{Methodology}

This study was conducted in Patna Medical College and Hospital. The source of population of the study was all elderly patients of Patna Medical College \& Hospital with chronic musculoskeletal pain disorder from May 1,2018 to July 30- 2018. The main Inclusion criteria was all patient $>65$ years old those registered for management of chronic musculoskeletal pain. Those who were registered between May 1, 2018 to July 30- 2018. The major exclusion criteria were All patient those age was $<65$ years old, those patients who were admitted in inpatient ward at the time of data collection, those patient who were with chronic musculoskeletal malignant pain and those patients with post-operative pain.

Data was collected by a nurses through retrospective by using semi structured questionnaires among elderly patients' chart those above 65 years old who were registered for management of chronic musculoskeletal pain in outpatient department in PMCH from May 1, 2018 to July 30- 2018. The clarity and completeness check-up of collected data was under taken after the actual data collection and data clearing was conducted.

\section{Result}

From a total of 131 chronic musculoskeletal pain management in elderly patient those registered at
Patna Medical College \& Hospital from May 1,2018 to July 30,2018 , of these $82(62.6 \%)$ were male and $49(37.4 \%)$ were female. This study shows that male were more affected with chronic musculoskeletal pain $82(62.6 \%)$ than female $49(37.4 \%)$ among this with gout arthritis $26(86.6 \%)$ were male and $4(13.3 \%)$ were female, Rheumatoid arthritis 46(58.9\%) were male and $32(41.1 \%)$ were female whereas with low back pain $10(43.5 \%)$ were male and $13(56.5 \%)$ were female.

Table 1: Socio-demographic characteristics of chronic musculoskeletal pain management

\begin{tabular}{|l|c|c|c|}
\hline \multicolumn{2}{|l|}{ Characteristics } & Frequency & Percent \\
\hline \multirow{3}{*}{ Sex } & Male & 82 & 62.6 \\
\cline { 2 - 4 } & Female & 49 & 37.4 \\
\hline \multirow{2}{*}{$\begin{array}{l}\text { Age } \\
\text { group }\end{array}$} & $65-75$ & 98 & 74.8 \\
\cline { 2 - 4 } & $76-85$ & 24 & 18.3 \\
\cline { 2 - 4 } & $\geq 86$ & 9 & 6.9 \\
\hline
\end{tabular}

Among 131 with chronic musculoskeletal pain the most prevalent was Rheumatoid arthritis $78(59.5 \%)$, gout arthritis 30(22.90\%) have moderate prevalence and low back pain 23 (17.6\%) was the least prevalent. Rheumatoid arthritis $46(58.9 \%)$ were male and $32(41.1 \%)$ were female whereas with low back pain $10(43.5 \%)$ were male and $13(56.5 \%)$ were female. (Figure 1)

Fig. 1: Prevalence of chronic musculoskeletal pain management

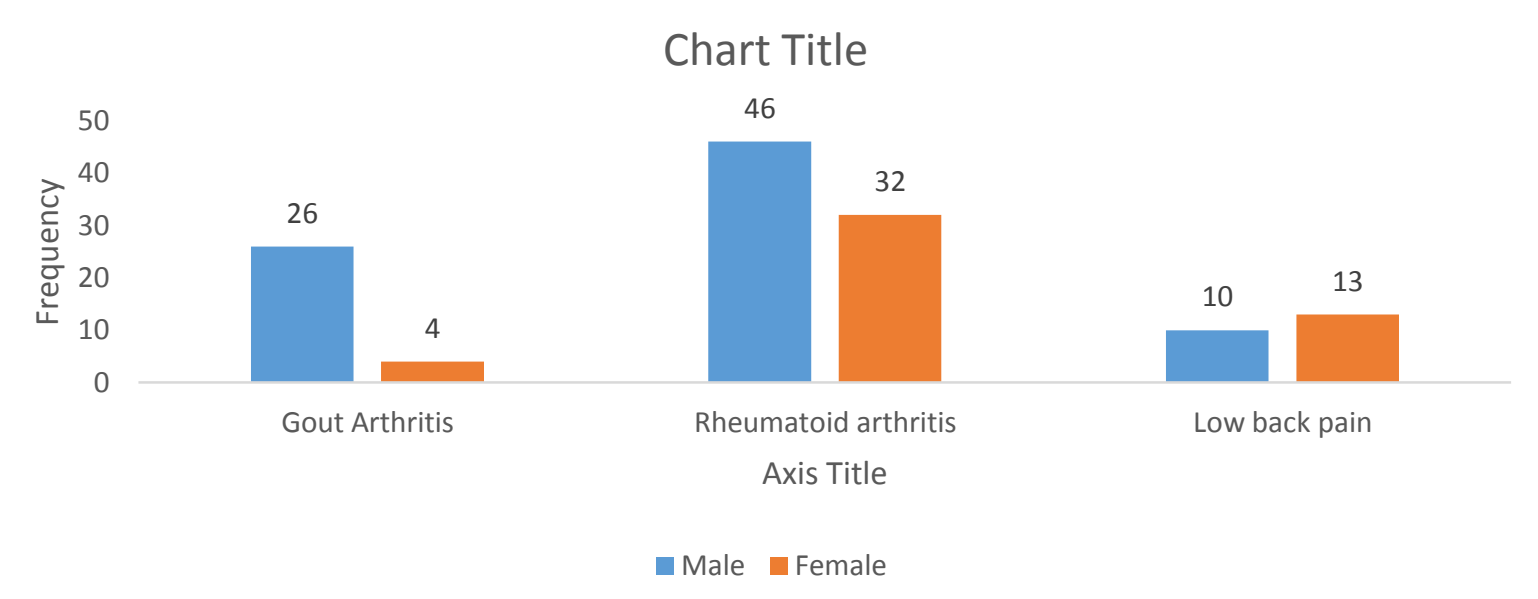

Among131patient 30(22.90\%) have gout arthritis, of these $10(33.3 \%)$ were treated with diclofenac IM minimum of 03 days and maximum of 05 days and Amitriptyline for 2 weeks duration of treatment, 15(50\%) were treated by Indomethacin for 14days duration of treatment and $5(16.7 \%)$ 
were treated with Tramadol IM for 5 days and prednisolone by tapering dose for minimum of 10

Fig 2: Drug therapy for Gout arthritis days and maximum of 15 days duration of treatment. (Figure 2)

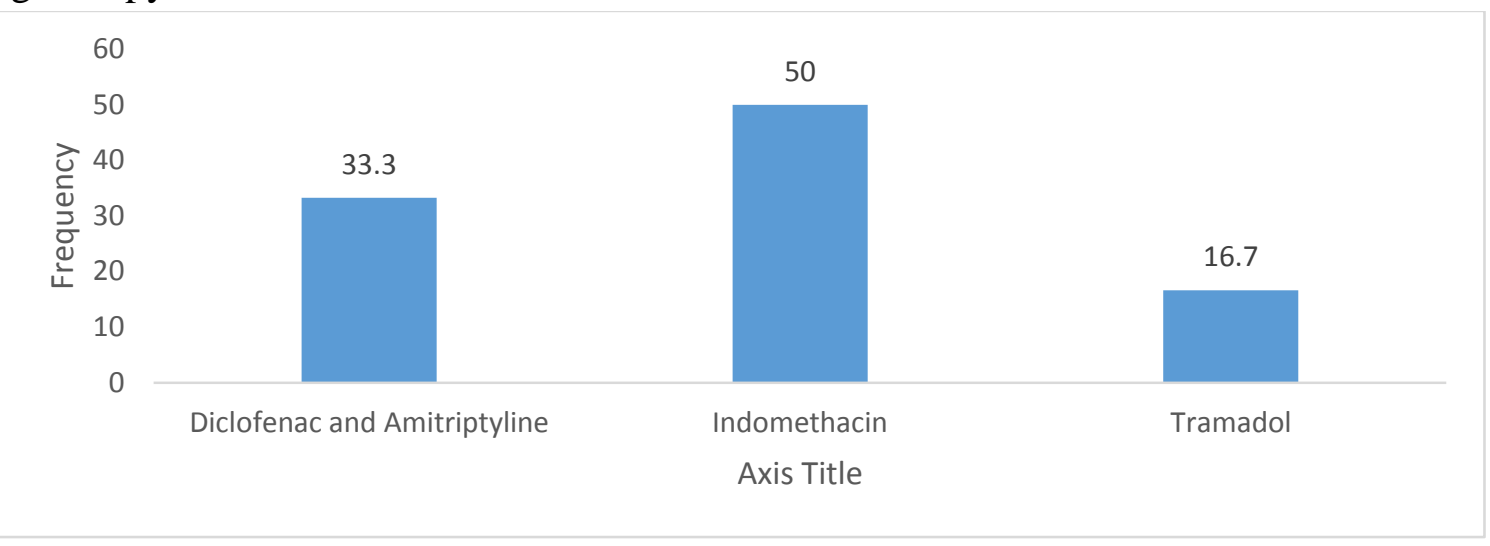

Out of 131 registered patients $78(59.5 \%)$ have Rheumatoid arthritis, of these $37(47.4 \%$ ) were treated with Indomethacin suppositories and po for 10 days and 14 days duration of treatment respectively, 23(29.5\%) were treated with Diclofenac IM and Dexamethasone IM concomitantly for minimum of 05 days and maximum of 07 days duration of treatment, 11(14.1\%)were treated with Ibuprofen po for minimum of 10days duration of treatment and maximum of 10days duration of treatment and $7(8.9 \%)$ were treated with Triamcinolone IM stat and Tramadol po for 05 days total duration of treatment. (Figure 3)

Fig 3: Drug therapy for Rheumatoid arthritis

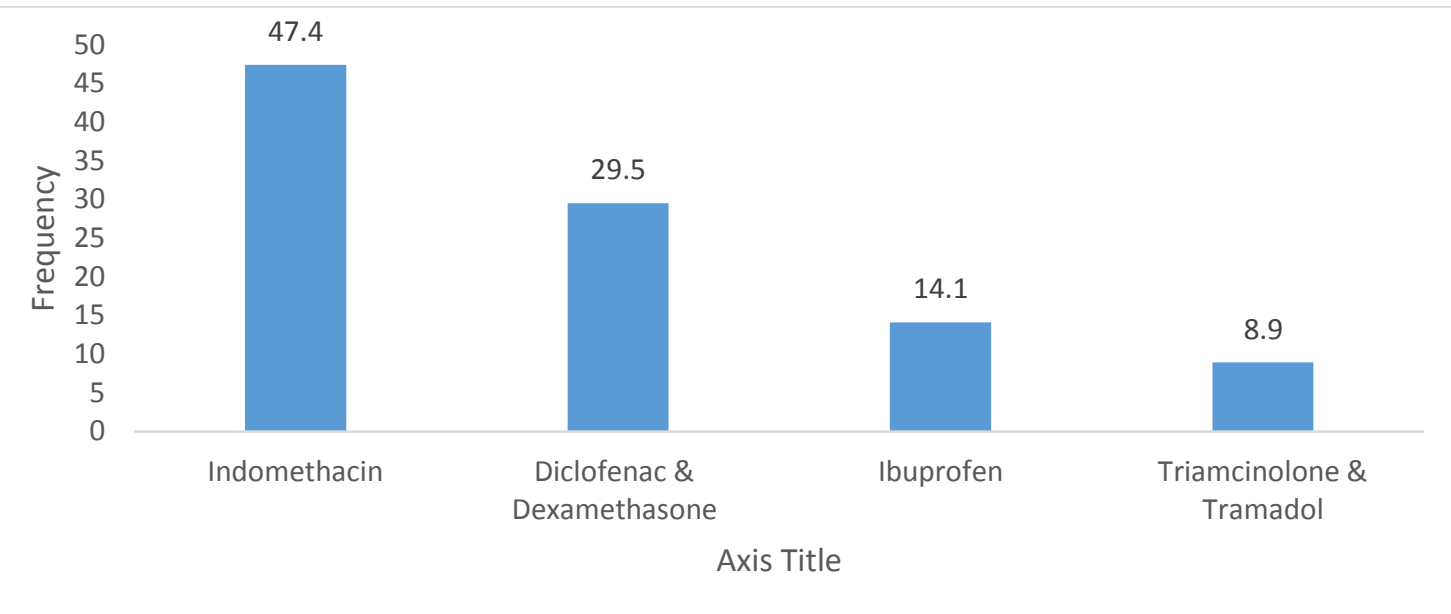

Among 131patients 23(17.5\%) have low back pain, of these $10(43.5 \%)$ were treated with Tramadol for minimum of 05days and maximum of 10days total duration of treatment, 7(30.3\%)were treated by Indomethacin for 1014days plus Amitriptyline for 2 weeks total duration of treatment, 4(17.4\%) were treated with Diclofenac IM plus Dexamethasone IM for 05 days and 2(8.7\%) were treated with Ibuprofen for minimum of 05 days and maximum of 10 days total duration of treatment. (Figure 4) 
Fig. 4: Drug therapy for low back pain

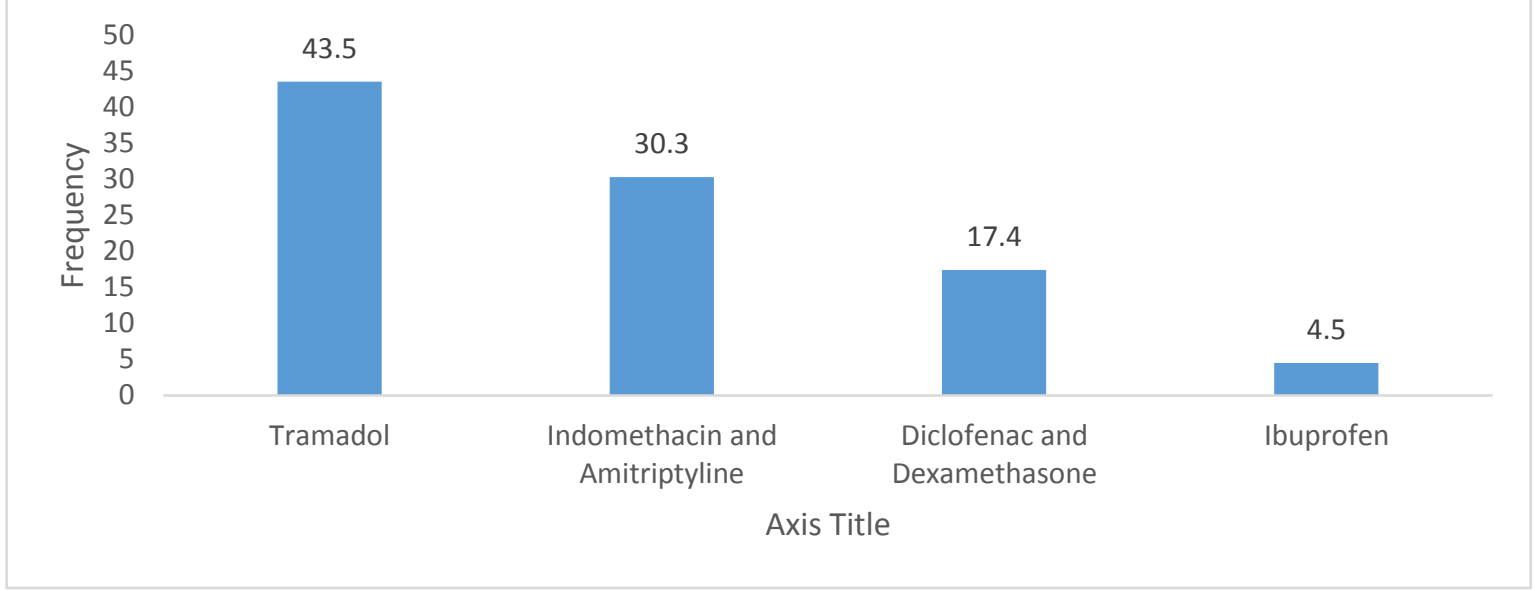

\section{Discussion}

As American Geriatrics Study Panel shows, the incidence and prevalence of chronic musculoskeletal pain generally rise with increasing age and higher among women. ${ }^{[16]}$ But the study conducted in Patna Medical College and Hospital from May 1,2018-July 30,2018, shows that the prevalence of chronic musculoskeletal pain in elderly was higher among men $82(62.6 \%)$ when compared with women $49(37.4 \%)$. In this assessment patient with gouty arthritis were treated by Diclofefenac, Amitriptyline, indomethacin, Tramadoland prednisolone as follow. From a total of 131patient 30(22.90\%) have gout arthritis, of these $10(33.3 \%)$ were treated with diclofenac IM minimum of 03 days and maximum of 05 days and amitriptyline po for 2 weeks duration of treatment, $15(50 \%)$ were treated by Indomethacin po for 14days duration of treatment and 5(16.7\%) were treated with Tramadol IM for 5 days and prednisolone po by tapering dose for minimum of 10 days and maximum of 15 days duration of treatment.

As study conducted by Joint Committee of the Medical Research Council of Australia suggested, patients treated with methotrexate have the highest rate of continued long-term therapy, and therefore most rheumatologists consider it the drug of choice. Disease-modifying drugs were effective as combination therapy for rheumatoid arthritis and whether the combinations studied had better efficacy than methotrexate alone. Conventional therapy for rheumatoid arthritis includes the administration of anti-inflammatory drugs, followed by disease-modifying ant rheumatic drugs such as methotrexate, hydroxychloroquine, sulfasalazine, and gold in patients with persistent active disease. ${ }^{[2]}$

\section{Conclusion}

This study shows, from chronic musculoskeletal pain, Rheumatoid arthritis was more prevalent78 (59.5\%) when compared with other type of chronic musculoskeletal pain in elderly while, low back pain is the least10(43.5\%) and gout arthritis $30(22.90 \%)$ have moderate prevalence and chronic musculoskeletal pain was more prevalent in male elderly patient $82(62.6 \%)$ when compared with female elderly patient $49(37.4 \%)$ as indicated from patient chart which were recorded from May 1,2018 to Jul 30,2018 at Patna Medical College \& Hospital. Since treating elderly patient need great precaution regarding drug metabolism and excretion so prior to prescribing NSAID, Steroid and other anti-pain to treat Chronic MSK pain management in elderly, pertinent lab finding such as Liver function test, Renal function test are essential.

\section{References}

1. Woolf AD, Akesson K. Understanding the burden of musculoskeletal conditions. The burden is huge and not reflected in national health priorities. BMJ, 2001; 322: 1079-80. 
2. The Consensus Document. The Bone and Joint Decade 2000-2010. Inaugural Meeting 17 and 18 April 1998. Acta Orthopaedica Scandinavica, 1998; 69: 67-86.

3. Woolf AD. The bone and joint decade 20002010. Annals of Rheumatic Disease, 2000; 59: 81 .

4. The burden of musculoskeletal diseases at the start of the new millennium. Report of a WHO scientific group. Geneva: World Health Organization, 2003. Technical Report Series, No. 919. Forthcoming.

5. Brage S, Nygard JF, Tellnes G. The gender gap in musculoskeletal-related long-term sickness absence in Norway. Scandinavian Journal of Social Medicine, 1998; 26:34-43.

6. Barbara St. Marie. Core Curriculum for pain management nursing. Second edition, 2012.

7. American Geriatric Society, Panel on persistent pain in older adults. J Am GeriatrSoc, 2002; 50: 205-224.

8. Jovey RD, Ennis J, Gardner-Nix J, Goldman B, Hays H, Lynch M et al, Use of opioid analgesics for the treatment of chronic non, 2003.

9. Parmelee PA, Smith B, Katz IR: Pain complaints and cognitive status among elderly institution residents. J Am GeriatrSoc, 1993; 41: 517-522.

10. Joint Commission on Accreditation of Healthcare Organizations (JCAHO): Implementing the new pain management standards. Oakbrook Terrace: JCAHO, 2012.

11. Tseng S, Wang R: Quality of life and related factors among elderly nursing home residents in southern Taiwan. Public Health Nursing, 2001; 18: 304-311.

12. Lavsky-Shulan M, Wallace RB, Kohout FJ: Prevalence and functional correlates of chronic musculoskeletal pain in the elderly: The Iowa 65+ Rural Health Study. J Am GeriatrSoc, 1985; 33: 23-28.

13. Podichetty VK,Mazaneck D, Biscup RS: Chronic nonmalignant musculoskeletal pain in older adults: clinical issues and opiod intervention. Post Grad Med Journal, 2003; 79: 627-633.

14. Davis MP, Horvitz HR: Demographics, assessment and management of pain in the elderly. Drugs and Aging, 2003; 20(1): 2357.

15. Chodosh J, Solomon D, Roth C, Chang J, MacLean C, Ferrell B, Shekelle P, Wenger $\mathrm{N}$ : The quality of medical care provided to vulnerable older patients with chronic pain. JAGS, 2004; 52: 756-76115.

16. American Geriatrics Study Panel: The American Geriatrics Society guidelines for the management of pain: implications in the LTC setting. 29th Annual Meeting of the AGS, 1998.

17. Papageorgiou AC, Croft PR, Ferry S, Jayson MIV, Silman AJ: Estimating the prevalence of low back pain in the general population. Evidence from the South Manchester Back Pain Survey. Spine, 1995; 20: 1889-1894.

18. Andersson GB. Epidemiological features of chronic low-back pain. Lancet, 1999; 354: 581.

19. Arnett FC, Edworthy SM, Bloch DA, McShane DJ,Fries JF, Cooper NS, et al. The AmericanRheumatism Association revised criteria for the classification of rheumatoid arthritis, 1987.

20. Kazis LE, Meenan RF, Anderson JJ. Pain in the rheumatic diseases. Investigation of a key health status component. Arthritis and Rheumatism, 1983; 26: 1017-22.

21. Coyte PC, Asche CV, Croxford R, Chan B. Gout arthritis oral and parenterral drug treatment in Canada. Arthritis Care and Research, 2008; 11: 315-25.

22. Brooks PM. MJA Practice Essentials Rheumatology. Sydney: Australian Medical Publishing Company Limited; 2010. 